https://doi.org/10.15407/ujpe63.8.733

T. ABEBE

Department of Physics, Jimma University

(P. O. Box 378, Jimma, Ethiopia; e-mail: tam1704@gmail.com)

\title{
ENHANCEMENT OF SQUEEZING \\ AND ENTANGLEMENT IN A NON-DEGENERATE THREE-LEVEL CASCADE LASER WITH COHERENTLY DRIVEN CAVITY
}

\begin{abstract}
We study a nondegenerate three-level cascade laser coupled to a two-mode vacuum reservoir, by employing the stochastic differential equations associated with the normal ordering. The amplification of the properties of squeezing and entanglement of the cavity light is investigated. We have found that there is an entanglement between the states of the light generated in the cavity, due to the strong correlation of the light emitted, when the atom decays from the top level to the bottom level via the intermediate one. We have also obtained that the two cavity modes are strongly entangled, and the degree of entanglement is directly related to the two-mode squeezing.
\end{abstract}

Keywords: atomic coherence, quadrature squeezing, entanglement.

\section{Introduction}

Quantum entanglement is the term given to the phenomena, whereby particles can be generated or interact in ways such that the quantum state of each particle cannot be described independently. In such cases, the system of particles is said to be entangled, and it is not proper to consider any of the individual particles in isolation from the others, but only as a single entangled state. Moreover, the entanglement is one of the most counter-intuitive aspects of the quantum world and an enigmatic powerful property. The generation and manipulation of the entanglement have attracted a great interest owing to their wide applications in quantum teleportation [1], quantum dense coding [2], quantum computation [3], quantum error correction [4], and quantum cryptography [5].

The efficiency of the quantum information processing highly depends on the degree of entanglement. Hence, it is desirable to generate a strongly entangled continuous variable state. Moreover, it has been shown that non-degenerate three-level cascade lasers can generate a macroscopic entangled state. In such lasers, the crucial role is played by the atomic coherence, which can be induced either by initially preparing the atoms in a coherent superposition of the top and bottom levels [6-10] or by the coupling

(C) T. ABEBE, 2018

ISSN 2071-0194. Ukr. J. Phys. 2018. Vol. 63, No. 8 of these levels by strong coherent light [11-17]. The injected coherent superposition creates a population transfer pathway, which is the basis for the correlated two-photon emission. Hence, a nondegenerate three-level laser is believed to be a source of a twomode squeezed light that is characterized by a strong correlation of two photons at two frequencies usually placed symmetrically at either side of the central frequency. The squeezing exists in the correlated state formed by the two modes, but it does not exist in single modes. In general, as a result of the strong correlation between the modes, a two-mode squeezed state violates certain classical inequalities and, hence, can be applied to preparing a Einstein-Podolsky-Rosen (EPR)-type entanglement [18].

Here, we will analyze a nondegenerate three-level cascade laser coupled to a two-mode vacuum reservoir via a single-port mirror. As shown in Fig. 1 for the nondegenerate cascade configuration, when the atom decays from level $|a\rangle$ to $|c\rangle$ via $|b\rangle$, two photons with different frequencies are generated. In this respect, we define a nondegenerate three-level cascade laser as a quantum optical system, in which nondegenerate three-level atoms, which are in a cascade configuration and initially prepared in a coherent superposition of the top and bottom levels, are injected at a constant rate into a cavity. Then we derive the pertinent master equation in the linear and adiabatic approxi- 
$+i \varepsilon\left(\hat{a}^{\dagger}-\hat{a}+\hat{b}^{\dagger}-\hat{b}\right)$

where $\varepsilon$, considered to be real and constant, is proportional to the amplitude of the driving coherent light, $g$ is a coupling constant, and $\hat{a}$ and $\hat{b}$ are, respectively, the annihilation operators for the two cavity modes.

In this paper, we take the initial state of a threelevel atom to be

$\left|\psi_{A}(0)\right\rangle=C_{a}(0)|a\rangle+C_{c}(0)|c\rangle$.

Hence, the initial density operator for a single atom has the form

$\hat{\rho}_{A}(0)=\rho_{a a}^{(0)}|a\rangle\left\langle a\left|+\rho_{a c}^{(0)}\right| a\right\rangle\left\langle c\left|+\rho_{c a}^{(0)}\right| c\right\rangle\langle a|+$

$+\rho_{c c}^{(0)}|c\rangle\langle c|$,

where $\rho_{a a}^{(0)}=\left|C_{a}\right|^{2}$ and $\rho_{c c}^{(0)}=\left|C_{c}\right|^{2}$ are, respectively, the probabilities for the atom to be initially in the upper and lower levels, and $\rho_{a c}^{(0)}=C_{a} C_{c}^{*}$ and $\rho_{c a}^{(0)}=$ $=C_{c} C_{a}^{*}$ represent the initial atomic coherence of the atom [20]. Using Eqs. (1) and (3) and employing the linear and adiabatic approximation schemes in the good cavity limit, we obtain the master equation for the cavity modes of the quantum optical system under consideration to be

$\frac{d}{d t} \hat{\rho}=\varepsilon\left[\hat{\rho} \hat{a}-\hat{a} \hat{\rho}+\hat{\rho} \hat{b}-\hat{b} \hat{\rho}+\hat{a}^{\dagger} \hat{\rho}-\hat{\rho} \hat{a}^{\dagger}+\hat{b}^{\dagger} \hat{\rho}-\hat{\rho} \hat{b}^{\dagger}\right]+$ $+\frac{\kappa}{2}\left[2 \hat{a} \hat{\rho} \hat{a}^{\dagger}-\hat{a}^{\dagger} \hat{a} \hat{\rho}-\hat{\rho} \hat{a}^{\dagger} \hat{a}\right]+\frac{A \rho_{a a}^{(0)}}{2}\left[2 \hat{a}^{\dagger} \hat{\rho} \hat{a}-\hat{a} \hat{a}^{\dagger} \hat{\rho}-\right.$ $\left.-\hat{\rho} \hat{a} \hat{a}^{\dagger}\right]+\frac{1}{2}\left(\kappa+A \rho_{c c}^{(0)}\right)\left[2 \hat{b} \hat{\rho} \hat{b}^{\dagger}-\hat{b}^{\dagger} \hat{b} \hat{\rho}-\hat{\rho} \hat{b}^{\dagger} \hat{b}\right]+$ $+\frac{A \rho_{a c}^{(0)}}{2}\left[\hat{\rho} \hat{b}^{\dagger} \hat{a}^{\dagger}+\hat{b}^{\dagger} \hat{a}^{\dagger} \hat{\rho}-2 \hat{a}^{\dagger} \hat{\rho} \hat{b}^{\dagger}\right]+$ $+\frac{A \rho_{c a}^{(0)}}{2}[\hat{a} \hat{b} \hat{\rho}+\hat{\rho} \hat{a} \hat{b}-2 \hat{b} \hat{\rho} \hat{a}]$, jected into a cavity at a constant rate $r_{a}$ and removed after a certain time $\tau$. The transitions between levels $|a\rangle$ and $|b\rangle$ and between levels $|b\rangle$ and $|c\rangle$ are dipoleallowed. However, the direct transition between levels $|a\rangle$ and $|c\rangle$ is dipole-forbidden. The interaction of the cavity modes with external driving fields and with a single three-level cascade atom is described, in the rotating wave approximation and in the interaction picture, by the Hamiltonian

$\hat{H}=i g\left[\hat{a}|a\rangle\langle b|-| b\rangle\left\langle a\left|\hat{a}^{\dagger}-\right| c\right\rangle\left\langle b\left|\hat{b}^{\dagger}+\hat{b}\right| b\right\rangle\langle c|\right]+$ where $A=\frac{2 r_{a} g^{2}}{\gamma^{2}}$ is the linear gain coefficient, $\gamma$ being the spontaneous atomic decay rate, and $\kappa$ is the cavity mode damping constant.

\section{Stochastic Differential Equations}

We now seek to obtain the stochastic differential equations associated with the normal ordering for the cavity mode variables. To this end, by applying 
Eq. (4) and the fact that $\frac{d}{d t}\langle\hat{A}\rangle=\operatorname{Tr}\left(\frac{d \hat{\rho}}{d t} \hat{A}\right)$, we can easily obtain

$$
\begin{aligned}
& \frac{d}{d t}\langle\hat{a}\rangle=-\frac{\mu}{2}\langle\hat{a}\rangle-\frac{A \rho_{a c}^{(0)}}{2}\left\langle\hat{b}^{\dagger}\right\rangle+\varepsilon, \\
& \frac{d}{d t}\langle\hat{b}\rangle=-\frac{\nu}{2}\langle\hat{b}\rangle+\frac{A \rho_{a c}^{(0)}}{2}\left\langle\hat{a}^{\dagger}\right\rangle+\varepsilon, \\
& \frac{d}{d t}\left\langle\hat{a}^{\dagger} \hat{a}\right\rangle=-\mu\left\langle\hat{a}^{\dagger} \hat{a}\right\rangle-\frac{A \rho_{a c}^{(0)}}{2}\left(\left\langle\hat{a}^{\dagger} \hat{b}^{\dagger}\right\rangle+\langle\hat{a} \hat{b}\rangle\right)+ \\
& +\varepsilon\left\langle\left(\hat{a}^{\dagger}\right\rangle+\langle\hat{a}\rangle\right)+A \rho_{a a}^{(0)}, \\
& \frac{d}{d t}\left\langle\hat{b}^{\dagger} \hat{b}\right\rangle=-\nu\left\langle\hat{b}^{\dagger} \hat{b}\right\rangle+\frac{A \rho_{a c}^{(0)}}{2}\left(\left\langle\hat{a}^{\dagger} \hat{b}^{\dagger}\right\rangle+\langle\hat{a} \hat{b}\rangle\right)+ \\
& +\varepsilon\left(\left\langle\hat{b}^{\dagger}\right\rangle+\langle\hat{b}\rangle\right), \\
& \frac{d}{d t}\langle\hat{a} \hat{b}\rangle=-\frac{\xi}{2}\langle\hat{a} \hat{b}\rangle-\frac{A \rho_{a c}^{(0)}}{2}\left(\left\langle\hat{b}^{\dagger} \hat{b}\right\rangle+\left\langle\hat{a}^{\dagger} \hat{a}\right\rangle-1\right)+ \\
& +\varepsilon(\langle\hat{a}\rangle+\langle\hat{b}\rangle),
\end{aligned}
$$

where $\mu=\kappa-A \rho_{a a}^{(0)}, \nu=\kappa+A \rho_{c c}^{(0)}$, and $\xi=2 \kappa+$ $A\left(\rho_{c c}^{(0)}-\rho_{a a}^{(0)}\right)$.

We note that all operators in Eqs. (5)-(9) are in the normal order. As a result, the corresponding expressions in terms of the $c$-number variables associated with the normal ordering can be put in the form

$$
\begin{aligned}
& \frac{d}{d t}\langle\alpha\rangle=-\frac{\mu}{2}\langle\alpha\rangle-\frac{A \rho_{a c}^{(0)}}{2}\left\langle\beta^{*}\right\rangle+\varepsilon \\
& \frac{d}{d t}\langle\beta\rangle=-\frac{\nu}{2}\langle\beta\rangle+\frac{A \rho_{a c}^{(0)}}{2}\left\langle\alpha^{*}\right\rangle+\varepsilon \\
& \frac{d}{d t}\left\langle\alpha^{*} \alpha\right\rangle=-\mu\left\langle\alpha^{*} \alpha\right\rangle-\frac{A \rho_{a c}^{(0)}}{2}\left(\left\langle\alpha^{*} \beta^{*}\right\rangle+\langle\alpha \beta\rangle\right)+ \\
& +\varepsilon\left\langle\left(\alpha^{*}\right\rangle+\langle\alpha\rangle\right)+A \rho_{a a}^{(0)}, \\
& \frac{d}{d t}\left\langle\beta^{*} \beta\right\rangle=-\nu\left\langle\beta^{*} \beta\right\rangle+\frac{A \rho_{a c}^{(0)}}{2}\left(\left\langle\alpha^{*} \beta^{*}\right\rangle+\langle\alpha \beta\rangle\right)+ \\
& +\varepsilon\left(\left\langle\beta^{*}\right\rangle+\langle\beta\rangle\right), \\
& \frac{d}{d t}\langle\alpha \beta\rangle=-\frac{\xi}{2}\langle\alpha \beta\rangle-\frac{A \rho_{a c}^{(0)}}{2}\left(\left\langle\beta^{*} \beta\right\rangle+\left\langle\alpha^{*} \alpha\right\rangle-1\right)+ \\
& +\varepsilon(\langle\alpha\rangle+\langle\beta\rangle)+\frac{A \rho_{a c}^{(0)}}{2} .
\end{aligned}
$$

On the basis of Eqs. (10) and (11), one can write

$$
\begin{aligned}
& \frac{d}{d t} \alpha(t)=-\frac{1}{2}\left(\kappa-A \rho_{a a}^{(0)}\right) \alpha(t)-\frac{A \rho_{a c}^{(0)}}{2} \beta^{*}(t)+ \\
& +\varepsilon+f_{\alpha}(t),
\end{aligned}
$$

$\frac{d}{d t} \beta^{*}(t)=-\frac{1}{2}\left(\kappa+A \rho_{c c}^{(0)}\right) \beta^{*}(t)-\frac{A \rho_{a c}^{(0)}}{2} \alpha(t)+$

$+\varepsilon+f_{\beta}^{*}(t)$,

where $f_{\alpha}(t)$ and $f_{\beta}^{*}(t)$ are the pertinent noise forces, whose properties remain to be determined. It is obvious that the expectation values of Eqs. (15) and (16) are identical to (10) and (11) provided that

$\left\langle f_{\alpha}(t)\right\rangle=\left\langle f_{\beta}^{*}(t)\right\rangle=0$.

Moreover, making use of (15) and (16), we can verify that

$$
\begin{aligned}
& \left\langle f_{\alpha}\left(t^{\prime}\right) f_{\alpha}^{*}(t)\right\rangle=A \rho_{a a}^{(0)} \delta\left(t-t^{\prime}\right), \\
& \left\langle f_{\alpha}\left(t^{\prime}\right) f_{\beta}(t)\right\rangle=\left\langle f_{\beta}\left(t^{\prime}\right) f_{\alpha}(t)\right\rangle=\frac{A \rho_{c a}^{(0)}}{2} \delta\left(t-t^{\prime}\right), \\
& \left\langle f_{\alpha}\left(t^{\prime}\right) f_{\alpha}(t)\right\rangle=\left\langle f_{\beta}\left(t^{\prime}\right) f_{\beta}(t)\right\rangle=0, \\
& \left\langle f_{\beta}^{*}\left(t^{\prime}\right) f_{\beta}(t)\right\rangle=\left\langle f_{\alpha}^{*}\left(t^{\prime}\right) f_{\beta}(t)\right\rangle=\left\langle f_{\alpha}^{*}(t) f_{\beta}\left(t^{\prime}\right)\right\rangle=0 .
\end{aligned}
$$

We note that (17)-(21) represent the mean and correlation properties of the noise forces.

It proves to be useful to introduce a parameter, which relates the probabilities of the atom to be in the upper and lower levels. We define a parameter $\eta$ such that

$\rho_{a a}^{(0)}=\frac{1-\eta}{2}$.

In view of the fact that

$\rho_{c c}^{(0)}=\frac{1+\eta}{2}$

and

$\left|\rho_{a c}^{(0)}\right|^{2}=\rho_{a a}^{(0)} \rho_{c c}^{(0)}$,

we easily find

$\rho_{a c}^{(0)}=\frac{1}{2} \sqrt{1-\eta^{2}}$

with $-1 \leq \eta \leq 1$. We note that the steady-state solutions Eqs. (10)-(14) are valid only for non-negative values of $\eta$, that is, $0 \leq \eta \leq 1$. Hence, with regard for $(22-25)$, we express (10) and (11) as

$\frac{d}{d t} \alpha(t)=-\left(\Gamma-\frac{A}{4}\right) \alpha(t)-\Delta \beta^{*}(t)+\varepsilon+f_{\alpha}(t)$, 
$\frac{d}{d t} \beta^{*}(t)=-\left(\Gamma+\frac{A}{4}\right) \beta^{*}(t)+\Delta \alpha(t)+\varepsilon+f_{\beta}^{*}(t)$,

where $\Gamma=(2 \kappa+A \eta) / 4$ and $\Delta=\frac{A \sqrt{1-\eta^{2}}}{4}$.

We realize that (26) and (27) are coupled differential equations. In order to solve these differential equations, we introduce a matrix equation of the form

$\frac{d}{d t} U(t)=-M U(t)+N(t)$,

in which

$U(t)=\left(\begin{array}{c}\alpha(t) \\ \beta^{*}(t)\end{array}\right)$,

$M=\left(\begin{array}{cc}\Gamma-\frac{A}{4} & \Delta \\ -\Delta & \Gamma+\frac{A}{4}\end{array}\right)$,

$N(t)=\left(\begin{array}{l}f_{\alpha}(t)+\varepsilon \\ f_{\beta}^{*}(t)+\varepsilon\end{array}\right)$.

Following the procedure described in Ref. [21], we obtain

$\alpha(t)=A_{+}(t) \alpha(0)+B_{+}(t) \beta^{*}(0)+F_{+}(t)+G_{+}(t)$,

$\beta^{*}(t)=A_{-}(t) \beta(0)+B_{-}(t) \alpha^{*}(0)+F_{-}(t)+G_{-}(t)$,

where

$$
\begin{aligned}
& A_{ \pm}(t)=\frac{1}{2 \eta}\left[(\eta \mp 1) e^{-(\Gamma+A \eta / 4) t}+(\eta \pm 1) e^{-(\kappa / 2) t}\right], \\
& B_{ \pm}(t)= \pm \frac{2 \Delta}{A \eta}\left[e^{-(\Gamma+A \eta / 4) t}-e^{-(\kappa / 2) t}\right], \\
& F_{ \pm}(t)=\frac{1}{2 \eta} \int_{0}^{t}\left[(\eta \mp 1) e^{-(\Gamma+A \eta / 4)\left(t-t^{\prime}\right)}+\right. \\
& \left.+(\eta \pm 1) e^{-[\kappa / 2]\left(t-t^{\prime}\right)}\right] f_{\alpha}\left(t^{\prime}\right) d t^{\prime} \pm \\
& \pm \frac{\varepsilon[4 \Delta \mp A(\eta \pm 1)]}{\kappa A \eta}\left(e^{-(\kappa / 2) t}-1\right), \\
& G_{ \pm}(t)= \pm \frac{2 \Delta}{A \eta} \int_{0}^{t}\left[e^{-[\Gamma+A \eta / 4]\left(t-t^{\prime}\right)}-e^{-[\kappa / 2]\left(t-t^{\prime}\right)}\right] \times \\
& \times f_{\beta}^{*}\left(t^{\prime}\right) d t^{\prime} \mp \frac{\varepsilon[4 \Delta \pm A(\eta \mp 1)]}{A \eta(4 \Gamma-\kappa)}\left(e^{-[\Gamma+A \eta / 4] t}-1\right) .
\end{aligned}
$$

One can be realize that a well-behaved solution of (28) exists in the steady state provided that $\eta>$ $>-\kappa / A$. Hence, $\eta=-\kappa / A$ can be interpreted as a threshold condition.

\section{Quadrature Variance}

Here, we seek to analyze the quadrature squeezing properties of the two-mode light in the cavity. The squeezing properties of the two-mode light in the cavity can be described by two quadrature operators defined as

$\hat{c}_{+}=\hat{c}^{\dagger}+\hat{c}$

and

$\hat{c}_{-}=i\left(\hat{c}^{\dagger}-\hat{c}\right)$,

where $\hat{c}=\frac{1}{\sqrt{2}}(\hat{a}+\hat{b})$. These quadrature operators satisfy the commutation relation $\left[\hat{c}_{+}, \hat{c}_{-}\right]=2 i$. On the basis of these definitions, a two-mode light is said to be in a two-mode squeezed state, if either $\Delta c_{+}^{2}<1$ or $\Delta c_{-}^{2}<1$. The variances of the quadrature operators can be expressed as

$\Delta c_{ \pm}^{2}=\left\langle\hat{c}_{ \pm}^{2}\right\rangle-\left\langle\hat{c}_{ \pm}\right\rangle^{2}$.

It is possible to express the variance of the quadrature operators (38) and (39) in terms of the $c$-number variables associated with the normal ordering, by taking the cavity modes to be initially in a two-mode vacuum state, as

$\Delta c_{ \pm}^{2}=1+\left\langle\alpha^{*}(t) \alpha(t)\right\rangle+\left\langle\beta^{*}(t) \beta(t)\right\rangle+2\left\langle\alpha^{*}(t) \beta(t)\right\rangle \mp$ $\mp \frac{1}{2}\left[\left(\left\langle\alpha^{*}(t)\right\rangle+\left\langle\beta^{*}(t)\right\rangle\right) \pm(\langle\alpha(t)\rangle+\langle\beta(t)\rangle)\right] \pm$

$\pm\left[\left\langle\alpha^{2}(t)\right\rangle+\left\langle\beta^{2}(t)\right\rangle+2\langle\alpha(t) \beta(t)\rangle\right]$.

Thus, using the steady-state solutions of (10)-(14), the steady-state quadrature variances are found to be

$$
\begin{aligned}
& \Delta c_{ \pm}^{2}=\frac{(2 \kappa+A(1+\eta))\left(2 \kappa+A \eta \pm A \sqrt{1-\eta^{2}}\right)}{2(2 \kappa+A \eta)(\kappa+A \eta)}+ \\
& +\frac{A^{2}\left(1-\eta^{2}\right)}{2(2 \kappa+A \eta)(\kappa+A \eta)} .
\end{aligned}
$$

We clearly see from Eq. (42) that the quadrature variances are independent of the parameter $\varepsilon$, which represents the driving coherent light. This shows that the driving coherent light has no effect on the degree of squeezing of the two-mode light. This is due to the fact that the external coherent light does not introduce an additional coherence to the system, which is

ISSN 2071-0194. Ukr. J. Phys. 2018. Vol. 63, No. 8 
believed to be the source of squeezing in three-level cascade lasers $[6-8,10]$.

In Fig. 2, we plot the variances of the squeezed quadratures $\Delta c_{-}^{2}$ versus $\eta$ as the parameter for different values of the linear gain coefficient. It is possible to see from this plots that the two-mode squeezing increases with the linear gain coefficient like Tesfa's work [20]. Moreover, the value of $\eta$, at which the maximum squeezing occurs, decreases to zero, as the linear gain coefficient increases. Therefore, the twomode squeezing can be optimized by choosing small values $\eta$ and large values of $A / \kappa$. For instance, for $A / \kappa=500$, the level of the squeezing is found to be $70 \%$ at $\eta=0.1$ and below the vacuum level.

\section{Entanglement Amplification}

Here, we seek to study the entanglement condition for two modes in the cavity. A pair of particles is taken to be entangled in quantum theory, if its states cannot be expressed as a product of the states of its individual constituents. The preparation and manipulation of these entangled states that have nonclassical and nonlocal properties lead to a better understanding of the basic quantum principles. In other words, if the density operator for the combined state cannot be described as a combination of the product density operators of the constituents,

$\hat{\rho} \neq \sum_{j} P_{j} \hat{\rho}_{j}^{(1)} \otimes \hat{\rho}_{j}^{(2)}$,

where $P_{j} \geq 0$, and $\sum_{j} P_{j}=1$ is set to ensure the normalization of the combined density of state. To study the properties of the entanglement produced by this quantum optical system, we need an entanglement criterion for the system. According to the criteria set by Duan et al. [19], a quantum state of the system is entangled provided that the sum of the variances of the two EPR-type operators $\hat{u}$ and $\hat{v}$ satisfies the condition

$(\Delta \hat{u})^{2}+(\Delta \hat{v})^{2}<2$,

where

$\hat{u}=\hat{x}_{a}-\hat{x}_{b}, \quad \hat{v}=\hat{p}_{a}+\hat{p}_{b}$,

with

$$
\begin{aligned}
& \hat{x}_{a}=\left(\hat{a}^{\dagger}+\hat{a}\right) / \sqrt{2}, \quad \hat{x}_{b}=\left(\hat{b}^{\dagger}+\hat{b}\right) / \sqrt{2}, \\
& \hat{p}_{a}=i\left(\hat{a}^{\dagger}-\hat{a}\right) / \sqrt{2}, \quad \hat{p}_{b}=i\left(\hat{b}^{\dagger}-\hat{b}\right) / \sqrt{2},
\end{aligned}
$$

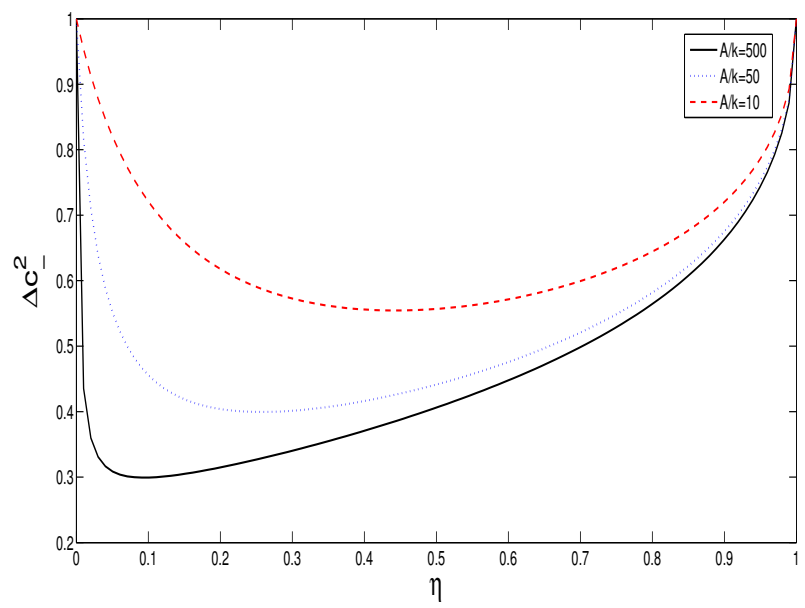

Fig. 2. Plots of the quadrature variance $\Delta c_{-}^{2}$ of the cavity radiation versus $\eta$ in the steady state for $\kappa=0.15$ and for different values of the linear gain coefficient

being the quadrature operators for the modes $\hat{a}$ and $\hat{b}$. The total variance of the operators $\hat{u}$ and $\hat{v}$ can be written as

$\Delta \hat{u}^{2}=\Delta \hat{v}^{2}=1+\left\langle\alpha^{*}(t) \alpha(t)\right\rangle+\left\langle\beta^{*}(t) \beta(t)\right\rangle+$

$+2\left[\left\langle\alpha^{*}(t) \beta(t)\right\rangle-\langle\alpha(t) \beta(t)\rangle\right]-$

$-\left[\left\langle\alpha^{2}(t)\right\rangle+\left\langle\beta^{2}(t)\right\rangle\right]$.

Thus, in view of Eq. (49) together with (41), the sum of the variances of $\hat{u}$ and $\hat{v}$ can be expressed as

$\Delta \hat{u}^{2}+\Delta \hat{v}^{2}=2 \Delta c_{-}^{2}$

where $\Delta c_{-}^{2}$ is given by Eq. (41). We see from this result that the degree of entanglement is directly proportional to the degree of squeezing of the two-mode light. Thus, with the aid of Eq. (42), we get

$$
\begin{aligned}
& \Delta \hat{u}^{2}+\Delta \hat{v}^{2}=\frac{(2 \kappa+A(1+\eta))\left(2 \kappa+A \eta \pm A \sqrt{1-\eta^{2}}\right)}{(2 \kappa+A \eta)(\kappa+A \eta)}+ \\
& +\frac{A^{2}\left(1-\eta^{2}\right)}{(2 \kappa+A \eta)(\kappa+A \eta)} .
\end{aligned}
$$

We immediately note that this particular entanglement measure is directly related to the two-mode squeezing. This direct relationship shows that, whenever there is a two-mode squeezing in the system, there will be entanglement in the system as well. It also follows that the degree of entanglement does not depend on the external driving coherent light. This 


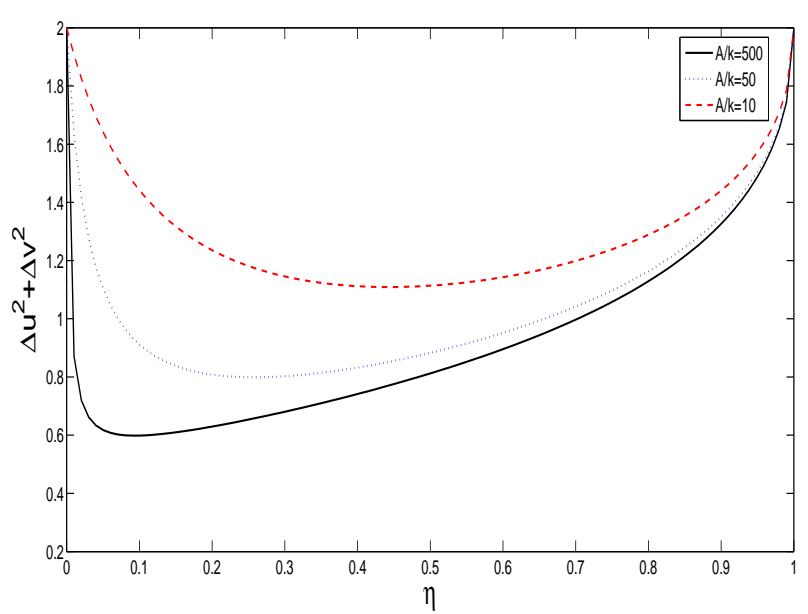

Fig. 3. Plots of $\Delta \hat{u}^{2}+\Delta \hat{v}^{2}$ of the two-mode light in the cavity in the steady state versus $\eta$ for $\kappa=0.15$ and for different values of the linear gain coefficient

is attributed to the fact that the coherent fields do not introduce an additional atomic coherence to the system, and the same is true in the case of squeezing. Using criterion (44) that a significant entanglement between the states of the light generated in the cavity of a nondegenerate three-level cascade laser can be manifested due to the strong correlation between the radiation emitted, when the atoms decay from the upper energy level to the lower via the intermediate energy level.

Based on criterion (44), we clearly see from Fig. 3 that the two states of the generated light are strongly entangled in the steady state. The entanglement disappears, when there is no atomic coherence, and it would be stronger for certain values of the atomic coherence for every value of the linear gain coefficient. It can easily be seen that the degree of entanglement increases with the rate, at which the atoms are injected into the cavity, $A$.

\section{Conclusion}

In this paper, we have studied the steady-state twomode squeezing and entanglement of the light produced by a nondegenerate three-level cascade laser coupled to a two-mode vacuum reservoir in the linear and adiabatic approximation schemes in the good cavity limit. It is found that the two-mode cavity radiation exhibits squeezing properties under certain conditions pertaining to the initial preparation of the superposition, where the degree of squeezing in- creases with the linear gain coefficient. In particular, the squeezing property exists, if the atoms are initially prepared in such a way that there are more atoms on the bottom level than on the upper level.

Moreover, we have also found that there is a significant entanglement between the states of the light generated in the cavity of a nondegenerate three-level cascade laser due to the strong correlation of the light emitted, when the atom decays from the top level to the bottom level via the intermediate level. The results obtained indicate that the two cavity modes are strongly entangled, and the degree of entanglement is directly related to the two-mode squeezing.

1. J.M. Liu, B.S. Shi, X.F. Fan, J.Li, G.C. Guo. Wigner function description of continuous variable entanglement swapping. J. Opt. B: Quant. Semiclass. Opt. 3, 189 (2001).

2. S.L. Braunstein, H.J. Kimble. Dense coding for continuous variables. Phys. Rev. A 61, 42302 (2000).

3. S. Lloyd, S.L. Braunstein. Quantum computation over continuous variables. Phys. Rev. Lett. 82, 1784 (1999).

4. S. L Braunstein. Quantum error correction for communication with linear optics. Nature 394, 47 (1998).

5. T.C. Ralph. Continuous variable quantum cryptography. Phys. Rev. A 61, 010302 (2000).

6. M.O. Scully, K. Wodkiewicz, M.S. Zubairy, J. Bergou, N. Lu, J. Meyer ter Vehn. Two-photon correlated-spontaneous-emission laser: Quantum noise quenching and squeezing Phys. Rev. Lett. 60, 1832 (1988).

7. K. Fesseha. Three-level laser dynamics with squeezed light. Phys. Rev. A 63, 033811 (2001).

8. N. Lu, F.X. Zhao, J. Bergou. Nonlinear theory of a twophoton correlated-spontaneous-emission laser: A coherently pumped two-level-two-photon laser. Phys. Rev. A 39 , 5189 (1989).

9. N. Lu, S.Y. Zhu. Quantum theory of two-photon correlated-spontaneous-emission lasers: Exact atom-field interaction Hamiltonian approach. Phys. Rev. A 40, 5735 (1989).

10. C.A. Blockley, D.F. Walls. Intensity fluctuations in a frequency down-conversion process with three-level atoms Phys. Rev. A 43, 5049 (1991).

11. N.A. Ansari, J. Gea-Banacloche, M.S. Zubairy. Phase-sensitive amplification in a three-level atomic system. Phys. Rev. A 41, 5179 (1990).

12. N.A. Ansari. Effect of atomic coherence on the second-and higher-order squeezing in a two-photon three-level cascade atomic system. Phys. Rev. A 48, 4686 (1993).

13. J. Anwar, M.S. Zubairy. Quantum-statistical properties of noise in a phase-sensitive linear amplifier. Phys. Rev. A 49, 481 (1994).

14. N.A. Ansari. Theory of a two-mode phase-sensitive amplifier. Phys. Rev. A 46, 1560 (1992).

ISSN 2071-0194. Ukr. J. Phys. 2018. Vol. 63, No. 8 
15. H. Xiong, M.O. Scully, M.S. Zubairy. Correlated spontaneous emission laser as an entanglement amplifier. Phys. Rev. Lett. 94, 023601 (2005).

16. C.M. Caves, B.L. Schumaker. New formalism for twophoton quantum optics. I. Quadrature phases and squeezed states. Phys. Rev. A 31, 3068 (1985).

17. C.J. Villas-Boas, M.H.Y. Moussa. One-step generation of high-quality squeezed and EPR states in cavity QED. Eur. Phys. J. D 32, 147 (2005).

18. A. Einstein, B. Podolsky, R. Rosen. Can quantummechanical description of physical reality be considered complete? Phys. Rev. 47, 777 (1935).

19. L.M. Duan, G. Giedke, J.I. Cirac, P. Zoller. Inseparability criterion for continuous variable systems. Phys. Rev. Lett. 84, 2722 (2000).

20. S. Tesfa. Entanglement amplification in a nondegenerate three-level cascade laser. Phys. Rev. A 74, 043816 (2006).

21. M.O. Scully, M.S. Zubairy. Quantum Optics (Cambridge Univ. Press, 1997).

Received 07.05.18
T. Абебе

ПОСИЛЕННЯ СТИСНЕННЯ

І ЗАПЛУТУВАННЯ У НЕВИРОДЖЕНОМУ

ТРИРІВНЕВОМУ КАСКАДНОМУ ЛАЗЕРІ

3 КОГЕРЕНТНО КЕРОВАНОЮ ПОРОЖНИНОЮ

$\mathrm{P}$ е $з$ ю м е

Розглянуто невироджений трирівневий каскадний лазер, пов'язаний $з$ двомодовим вакуумним резервуаром, застосовуючи стохастичні диференціальні рівняння, що відповідають нормальному упорядкуванню. Вивчено посилення стиснення і заплутування порожнинної моди світла. Показано, що є заплутування між станами світла, яке генерується в порожнині завдяки сильній кореляції світла, що випромінює атом при переході з верхнього на нижній рівень через проміжний. Знайдено, що дві порожнинні моди сильно заплутані, і ступінь заплутування безпосередньо пов'язана 3 двомодовим стисненням. 